\title{
Dislodged Gastrostomy Tube in a Delirious Patient with Advanced Laryngeal Cancer
}

\author{
Mitchell L, Asimakopoulos P, Shakeel $M^{*}$ and Ah-See KW \\ Department of Otolaryngology-Head and Neck Surgery, Aberdeen Royal Infirmary, Aberdeen, United \\ Kingdom
}

\begin{abstract}
Introduction: Delirium is common in hospitalised patients and is often multifactorial. Patients with advanced oropharyngeal malignancy are at particular risk because of compromised airway contributing to hypoxia.
\end{abstract}

Objective: To present a case of a patient who removed her radiologically inserted gastrostomy tube by pulling it out highlighting the importance of observation and monitoring of mental state in patients at high risk of developing delirium.

Case Report: A 71-year-old female patient undergoing radiotherapy for head and neck cancer received a radiologically inserted gastrostomy (RIG) tube due to dysphagia and high risk of aspiration. Previous to this, it was necessary to have a radiologically guided nasogastric tube (NGT) inserted due to tumour related anatomical distortion in the laryngopharynx. This was pulled out or fell out on multiple occasions. On the first postoperative day after RIG tube insertion, nursing staff found the patient in her room with the tubing cut and RIG removed including the bumper.

\begin{abstract}
Conclusion: This case highlights the issues surrounding fluctuation of cognition in elderly cancer patients and how this could potentially lead to serious complications after invasive procedures. Patients should be closely monitored after undergoing such procedures and signs of delirium identified early to prevent possible significant morbidity or mortality.
\end{abstract}

\section{Keywords}

Delirium, Head and Neck cancer, Patient, RIG tube, Monitoring

\section{Introduction}

In patients with head and neck malignancy malnutrition is common as a result of the cancer site, the disease process and the treatment methods frequently used [1]. The result of poor nutrition and unintentional weight loss in these patients during and after treatment can lead to worse treatment outcomes and an increase in morbidity [2]. To try and optimise nutrition in these patients often feeding via nasogastric tube or percutaneous endoscopic gastrostomy (PEG) is used. In patients where the tumour size or position would make endoscopic gastrostomy difficult, a radiologically inserted gastrostomy (RIG) may be considered. Both RIG and PEG procedures are associated with a risk of morbidity and mortality. The significant complications include peritonitis [3], tube displacement and pneumoperitoneum [4]. A meta-analysis suggests that major complication rates are higher in RIG than percutaneous gastrostomy [5]. More specifically related to this case report, one of the severe complications more commonly associated with RIG compared to PEG was accidental removal of the feeding tube [6]. Patients with cancer are at risk of developing delirium due to many factors such as pain, medications, radiation treatment or surgery [7]. The multifactorial aetiology of delirium in these patients makes management of complications from treatment extremely challenging. It has been demonstrated that surgical tracheostomy is an independent risk factor for delirium in head and neck cancer patients [8] and there are other contributing factors in this case study including nutritional status, age and co-morbidities.

\section{Case Report}

A 77-year-old Caucasian female with history of smoking and drinking was admitted to our department with high suspicion of laryngeal malignancy after flexible nasal laryngoscopy

*Corresponding author: Mr. Muhammad Shakeel, Consultant otolaryngologist-head and neck surgeon, Otolaryngology-Head and Neck surgery, ward 210, ARI, Aberdeen, AB25 2ZN, United Kingdom, Tel: 00441224552100, Fax: 00441224554569, E-mail: drshakeel@doctors.org.uk

Accepted: February 13, 2021

Published online: February 15, 2021

Citation: Mitchell L, Asimakopoulos P, Shakeel M, et al. (2021) Dislodged Gastrostomy Tube in a Delirious Patient with Advanced Laryngeal Cancer. Otolaryngol Res Rev 4(1):69-71 
in the clinic confirmed the presence of a large supraglottic tumour involving the right epiglottis and aryepiglottic fold. The purpose of the admission was to assess her nutritional status and arrange cross sectional imaging followed by examination under anaesthesia with biopsy. Due to difficulties with awake fibre optic intubation the decision was made to proceed with tracheostomy under local anaesthetic. The tumour was biopsied trans-orally once the airway was secured. The tumour was staged as T4a N3b M0 moderately differentiated squamous cell carcinoma. Following discussion at the regional multidisciplinary team meeting, the patient was offered radical radiotherapy as she was not medically fit to undergo total laryngectomy.

Prior to this admission she was fully independent and lived alone. Significant past medical history included two previous myocardial infarctions, transient ischaemic attack and ischaemic heart disease. There were no known previous concerns regarding her cognitive function.

During her admission she required multiple nasogastric tubes to be inserted under flexible nasal laryngoscopy guidance due to the size and location of her tumour distorting the laryngopharyngeal anatomy. The NG tubes fell out on multiple occasions' particularity overnight. Following the decision to proceed with radical radiotherapy, we aimed to establish a more permanent nutrition route. We proceeded with insertion of radiological guided gastrostomy which was carried out successfully without any immediate complications. Unfortunately, one day after insertion the patient cut the tube with scissors and pulled out the external gastrostomy tube and bumper.

The patient had to be kept nil by mouth until a safe feeding route could be established. A week of total parenteral nutrition (TPN) was needed before re-commencing nasogastric feeding. There were no complications from the removal of the RIG and she remained clinically well while undergoing radiotherapy.

\section{Discussion}

This case highlights the vulnerability of cancer patients to delirium and the subsequent risks when they undergo invasive procedures. Although it is very important in head and neck cancer patients to establish a permanent feeding route to allow for uninterrupted treatment the morbidity associated is not negligible and this must be considered on an individual basis. For head and neck cancer patients it is still unclear on what is the optimum technique for gastrostomy placement and the morbidity remains higher in this patient demographic [5]. Although complication rates are higher with RIG compared to PEG, including accidental tube removal [6], the mechanism of this is not clear. It would be beneficial for future studies to ascertain in how many cases of accidental tube removal delirium may have been a contributing factor. For this reason the patients and families should be counselled before the procedure thoroughly to ensure they understand this additional risk. The method of removal of the RIG in this case by a patient cutting the tube has been observed in other patient case studies in relation to NG tubes [9] suggesting that closer monitoring of the environment of vulnerable patients may be needed including ensuring sharp objects are not near a patient's bed space. It has been demonstrated that cancer patients without any signs of delirium on admission are highly susceptible to developing it [10]. Post-operative delirium is associated with many complications including prolonged hospital stay, higher care costs and higher risk of institutionalisation on discharge [11]. In this case the patient has multiple factors which could have contributed to her confusional state and subsequent self removal of the RIG tube. These include the procedure itself, her advanced age, unfamiliar environment, sedative medication used during the procedure and an element of intermittent mild hypoxia due to blockage of tracheostomy with secretions. There was potentially a predictor of risk that the patient could interfere with the RIG tube as multiple NG tubes had been dislodged previously with no clear cause. It is recommended that there is increased monitoring and assessment of patients who are vulnerable to developing post-operative delirium [12]. The Scottish Intercollegiate Guideline Network (SIGN) recommends using the 4AT tool to screen for delirium to allow rapid identification in changes in mental state [13]. After identifying a patient is suffering from delirium, they should have a prompt pharmacological review and physical exam to exclude serious contributing factors such as hypoxia or hypoglycaemia [13]. The next step in managing patients with delirium involves optimising their environment and medications, providing consistent supportive nursing and good communication with the patient and their family. Nursing of this patient was performed in a single room which makes supervision more challenging. If staffing capacity does not allow for one-to-one nursing, monitoring vulnerable patients in a close observation unit or in a multiple bed room for signs of acute confusion is preferred. This approach could lead to a more judicious medical review and potentially prevent complications such as that seen in our patient. To prevent post-operative delirium most guidelines recommend preoperative cognitive screening, non-pharmacological delirium prevention methods and avoidance of deliriogenic medications [14]. In practice this would involve a low threshold for suspecting a patient may be at risk and making sure everyone involved in caring for the patient monitors them closely for changes in cognitive state and escalates changes promptly to the medical team for review. Adequate postoperative analgesia is important as pain itself can cause delirium but making sure that the medications prescribed are not in themselves worsening cognition. Regular pain reviews can be utilised to allow for analgesia to be rationalised appropriately in the hours and days after a procedure.

Fortunately, in this case there does not appear to have been any serious complications which can occur after RIG insertion such as pneumoperitoneum or perforation [15]. It did however expose the patient to risks including treatment delay and need for total parenteral nutrition. It has been shown that an increased waiting time for radiotherapy increases the risk of local recurrence of disease [16] so for this reason alone it is essential that any delays are prevented where possible in order to promote the best patient outcomes. There must also be the consideration of additional costs incurred by the health service as a result of having to insert a PICC line and commence TPN leading to a prolonged hospital stay. 


\section{Conclusion}

Hospitalised patients with cancer are overall at a high risk of developing a delirium and they should be monitored closely and regularly reviewed. All members of the multidisciplinary team should be involved in identifying delirium but also minimising the risk of this developing. In high risk patients every effort should be made to make their environment safe including removing sharp items which could cause harm to the patient or healthcare staff. In patients with head and neck cancer the risks associated with gastrostomy insertion is higher so careful consideration must be given to each individual in order to weigh up the risks against the potential benefits.

\section{Recommendations}

Pre-operative screening for patients undergoing head and neck cancer surgery to identify those at risk and allow contributing factors to be minimised.

Optimisation of patient environment and multidisciplinary awareness of hazards to delirious patient such as sharp objects in the room.

Using the 4AT tool regularly in post-operative head and neck cancer patients for early identification of delirium.

\section{Conflict of Interest}

None declared.

\section{References}

1. Ackerman D, Laszlo M, Provisor A, et al. (2018) Nutrition management for the head and neck cancer patient. Cancer Treat Res174:187-208.

2. Talwar B, Donnelly R, Skelly R, et al. (2016) Nutritional management in head and neck cancer: United Kingdom national multidisciplinary guidelines. J Laryngol Otol 130: S32-S40.

3. Nalagatla S, Shakeel M, Vallamkondu V, et al. (2014) Complication of percutaneous endoscopic gastrostomy (PEG) necessitating partial gastrectomy. Austin J Otolaryngol 1: 1022.

4. Potack JZ, Chokhavatia S (2008) Complications of and controversies associated with percutaneous endoscopic gastrostomy: Report of a case and literature review. Medscape J Med 10:142.
5. Grant D, Bradley P, Pothier D, et al. (2009) Complications following gastrostomy tube insertion in patients with head and neck cancer: A prospective multi-institution study systematic review and meta-analysis. Clin Otolaryngol 34: 103-112.

6. McAllister P, Maclver C, Wales C, et al. (2013) Gastrostomy insertion in head and neck cancer patients: $A 3$ year review of insertion method and complication rates. Br J Oral Maxillofac Surg 51: 714-718.

7. Edelstein A, Yesne A (2017) Diagnosing and managing delirium in cancer patients. Oncology (Williston Park) 31: 686-692.

8. Zhu Y, Wang G, Liu S, et al. (2017) Risk factors for postoperative delirium in patients undergoing major head and neck cancer surgery: A meta-analysis. Jpn J Clin Oncol 47: 505-511.

9. Sankar V, Shakeel M, Keh S, et al. (2012) A case of a 'lost' nasogastric tube. J Laryngol \& Otol 126: 1296-1298.

10. El Majzoub I, Abunafeesa H, Cheaito R, et al. (2019) Management of altered mental status and delirium in cancer patients. Ann of Palliative Med 8: 728-739.

11. Hughes CG, Boncyk CS, Culley DJ, et al. (2020) American society for enhanced recovery and perioperative quality initiative joint consensus statement on postoperative delirium prevention. Anesth Analg 130: 1572-1590.

12. Korc GB, Root JC, Alici Y (2015) Prevention of post-operative delirium in older patients with cancer undergoing surgery. J Geriatr Oncol 6: 60-69.

13. Soiza RL, Phyo K (2019) The Scottish intercollegiate guidelines network (SIGN) 157: Guidelines on risk reduction and management of delirium. Medicina (Kaunas) 55: 491.

14. Curtis MS, Forman NA, Donovan AL, et al. (2020) Postoperative delirium: Why, what, and how to confront it at your institution. Curr Opin Anaesthesiol 33: 668-673.

15. Abd RT, Sharratt C, Tinker M, et al. (2014) PTH-132 gastrostomy insertions: Is it all about choosing the right patients? A comparison between percutaneous endoscopic gastrostomy (peg) and radiologically inserted gastrostomy (rig) indications complications and mortality rates. Gut 63: A2692-A270.

16. Chen Z, King W, Pearcey R, et al. (2008) The relationship between waiting time for radiotherapy and clinical outcomes: A systematic review of the literature. Radiother Oncol 87: 3-16.

DOI: $10.36959 / 926 / 556$

Copyright: (C) 2021 Mitchell L, et al. This is an open-access article distributed under the terms of the Creative Commons Attribution License, which permits unrestricted use, distribution, and reproduction in any medium, provided the original author and source are credited. 